\title{
The Expression Pattern of $\beta$-Catenin in Mesothelial Proliferative Lesions and Its Diagnostic Utilities
}

\author{
Yiran Dai, M.D., ${ }^{1 *}$ Carlos W.M. Bedrossian, M.D., FIAC, ${ }^{2}$
} and Claire W. Michael, M.D. ${ }^{1}$

\begin{abstract}
$\beta$-Catenin is a component of the E-cadherin-catenin cell adhesion complex. It plays an important role in the Wnt/wg pathway, which conveys critical signals for cell proliferation and transformation. The $\beta$-catenin mutation is an important event in the progression of a number of malignancies. In this study, we evaluated the immunohistochemical (IHC) pattern of $\beta$-catenin in a spectrum of mesothelial lesions. Sixty-five formalin-fixed, paraffin-embedded blocks from 54 serous effusions and 11 pleural biopsies were examined. These cases consisted of 33 invasive mesotheliomas, 9 early mesotheliomas (with negative radiologic finding), so-called mesotheliomas in situ, and 23 reactive mesothelial proliferations. A distinct membranous and/or submembranous staining pattern was seen in 23 cases with normal and reactive mesothelium. In contrast, reduced membranous and/or submembranous antibody staining and markedly increased ectopic cytoplasmic and nuclear staining was seen in 26 cases of 33 mesotheliomas. Seven of 9 early mesotheliomas showed increased ectopic cytoplasmic andlor nuclear stain. On the basis of our findings, identification of $\beta$-catenin staining pattern offers a useful marker in the diagnosis of mesothelial lesions and may help identify neoplastic change. Diagn. Cytopathol. 2005;33:320-324. @ 2005 Wiley-Liss, Inc.
\end{abstract}

Key Words: mesothelioma; mesothelial cells; $\beta$-catenin; pleural fluid

Malignant mesothelioma is a rare and highly aggressive neoplasm. Within the last $50 \mathrm{yr}$, enormous interest has been generated by the rising incidence of this tumor, particularly after the recognition of asbestos as a main causative agent. Recently, as clinical management of mesothelioma develops, innovative therapies such as the use of targeted cytokines and gene therapy in early stage patients require early diagnosis of malignant mesothelioma by fluid cytology or small biopsy. ${ }^{1}$ However, a definitive diagnosis of malignancy, enabling prompt therapeutic decisions, is not always possible to obtain by conventional

\footnotetext{
${ }^{1}$ Department of Pathology, University of Michigan, Ann Arbor, Michigan

${ }^{2}$ Norwegian American Hospital, Chicago, Illinois

*Correspondence to: Yiran Dai, M.D. University of Michigan Hospital, Department of Pathology, 1500 E. Medical Center Drive, Room 2G332/Box 0054, Ann Arbor, MI 48105. E-mail: daiy@med.umich.edu

Received 23 February 2005; Accepted 25 March 2005

DOI 10.1002/dc.20281

Published online in Wiley InterScience (www.interscience.wiley.com).
}

fluid cytology and/or biopsy examination. Reactive mesothelial hyperplasia sometimes mimic malignancy, whereas the morphologic alterations in malignancy are often quite subtle and frequently pose diagnostic problems. ${ }^{2}$ Therefore, there is a pressing need for markers that can reliably facilitate distinguishing malignant mesothelioma from benign proliferative mesothelium. $\beta$-Catenin is a multifunctional protein. It mediates cell-to-cell adhesions together with the transmembrane glycoprotein E-cadherin. It is also an oncoprotein that can lead to carcinogenesis when the adenomatous polyposis coli (APC)/ $\beta$-catenin/ T-cell factor (Tcf) signal transduction pathway is disrupted. ${ }^{3,4}$ In the normal cells membrane-binding $\beta$-catenin is positive, but intracytoplasmic and nuclear $\beta$-catenin are not detectable by immunohistochemical (IHC) stain resulting in a distinct membranous staining. Recent studies have showed that accumulation of intracytoplasmic and nuclear $\beta$-catenin is associated with malignant transformation and unchecked cell proliferation of a wide range of human neoplasms. ${ }^{5} \beta$-Catenin mutation, to our knowledge, have not been reported in mesothelial lesions. We studied the IHC expression pattern of $\beta$-catenin in a spectrum of mesothelial lesions to determine its clinical utility.

\section{Materials and Methods}

\section{Specimens}

Sixty-five formalin-fixed, paraffin-embedded blocks from 54 serous effusions and 11 pleural biopsies were collected during a 10-yr period from the University of Michigan Hospitals and Northwestern University Hospital. These cases consisted of 33 invasive Mesotheliomas (26 epithelial type, 5 sarcomatous type, and 2 poorly differentiated type), 9 early mesotheliomas (so-called mesothelioma in situ), and 23 reactive mesothelial lesions including 4 cases with florid papillary hyperplasia (with extensive papillary/ solid clusters). Early mesothelioma is defined according to the criteria outlined by Whitaker et al.: (1) At the time of work up, the patient had no gross tumor seen either at open thoracotomy or pleuroscopy with the exception of 
minute sand grain-like spots or focal pleural thickening, (2) No lesion was identified radiologically, and (3) The diagnosis of malignant mesothelioma was established at some stage in the development of the disease either on a cytologic or histologic sample. ${ }^{6}$

\section{IHC Analysis of $\beta$-Catenin}

Five-micron sections of formalin-fixed, paraffin-embedded tissue were mounted on Probe-On slides (Fisher Scientific, Itasca, IL), deparaffinized in xylene, and then rehydrated into distilled water through graded alcohols. Antigen retrieval was enhanced by microwaving the slides in citrate buffer (pH 6.0, Biogenex, San Ramon, CA) for $10 \mathrm{~min}$. Endogenous peroxidase activity was quenched by incubation with $3 \%$ hydrogen peroxide in methanol, and then blocked with $1.5 \%$ normal horse serum for $1 \mathrm{hr}$. Sections were incubated with a mouse monoclonal anti- $\beta$-catenin antibody (C19220, Transduction Laboratories, Lexington, $\mathrm{KY}$ ) at a dilution of 1:500 overnight at $4^{\circ} \mathrm{C}$. Slides were washed with PBS, and then incubated with a biotinylated horse anti-mouse secondary antibody for $30 \mathrm{~min}$ at room temperature. Antigen-antibody complexes were detected with the avidin-biotin peroxidase method, using 3,3'-diaminobenzidine as a chromogenic substrate (Vectastain aneurysmal bone cyst (ABC) kit, Vector Laboratories, Burlingame, CA). Immunostained sections were lightly counter-stained with hematoxylin, then examined by light microscopy.

\section{Scoring}

Immunostaining was scored on a three tiered scale for intensity $(1+$, weak; $2+$, moderate; $3+$, strong) in the mesothelial cell nuclei, cytoplasm, and cell membranes. Scoring was done without knowledge of the diagnosis by two pathologists separately. Reproducibility of scoring was $>85 \%$ both within and between scorers.

\section{Results}

\section{Normal and Reactive Mesothelium}

A distinct membranous and/or submembranous staining of $\beta$-catenin was seen in $87 \%(20 / 23)$ of patients with normal and reactive mesothelioma (Fig. C-1, Table I). Only a faint cytoplasmic staining was observed in 3 cases of florid reactive mesothelium, but no nuclear expression of $\beta$-catenin was present.

\section{Invasive Mesothelioma}

The fraction of cases showing membranous and/or submembranous $\beta$-catenin staining was significantly lower in $21 \%(7 / 33)$ cases of invasive mesotheliomas. In contrast, markedly increased ectopic cytoplasmic and/or nuclear staining were seen in $79 \%$ (26/33) cases of invasive mesotheliomas $(80 \%(21 / 26)$ epithelial type (Figs. C-2 and C3), $80 \%(4 / 5)$ sarcomatous type (Fig. C-4), and 50\% (1/2) poorly differentiated type). Interestingly, the intensity of cytoplasmic and nuclear stain is significantly increased in the deep invasive portion of the tumor (Fig. C-5).

\section{Early Mesothelioma}

The majority of early mesotheliomas 78\% (7/9 cases) showed increased ectopic cytoplasmic and/or nuclear staining (Figs. C-6 and C-7). Membranous/submembranous staining was observed only in the remaining two cases.

\section{Discussion}

As innovative therapies bring the hope to the patients with early stage mesothelioma, the demand for an early, accurate diagnosis of mesothelioma became more pressing. However, the current approach for diagnosis of malignant mesothelioma is still mainly dependent on clinical and radiologic information in conjunction with pathologic tests. The common specimens used for pathologic examination are transthoracic biopsy and effusion cytology. Although transthoracic biopsy is considered to have a higher accuracy in diagnosing mesothelial lesions than thoracentesis, the procedure has some disadvantages. The morbidity is higher, the risk of spreading neoplastic cells along the surgical site was reported, and it is difficult to locate a small or superficial lesion. In comparison with biopsy, cytological examination of effusion fluid is more efficient and safe. This renders cytological examination of effusion as the preferred choice for establishing the diagnosis, particularly in those cases of early mesothelioma. Many morphologic criteria have been proposed for the diagnosis of malignant mesothelioma and separating it from adenocarcinoma (ACA). ${ }^{8,9}$ However, the greatest difficulty is in differential diagnosis of mesothelioma from benign mesothelial hyperplasia. ${ }^{10}$ On the basis of the morphologic criteria, the following three features are considered to have diagnostic value in tissue sections: presence or absence of invasion, the extent of proliferation, and cytological atypia. Because of the considerable morphologic overlaps between well-differentiated mesothelioma and mesothelial hyperplasia, ${ }^{11}$ none of these features is diagnostic. Therefore, a biologic marker is needed for facilitating the differential diagnosis.

Several recent studies revealed that $\beta$-catenin is a useful marker of neoplastic cell transformation. ${ }^{12} \beta$-catenin has several important functions. One of the biologic functions of $\beta$-catenin is cell-to-cell adhesion. In the adherence junctions of epithelial cells, the cytoplasmic domain of E-cadherin organizes a peripheral protein complex, including a-catenin, b-catenin, and r-catenin, that is necessary for adhesion to occur. The role of b-catenin in the linkage between a-catenin and E-cadherin in this complex is probably regulated by epidermal growth factor-mediated tyrosine phosphorylation of the $\beta$-catenin. Reduced 


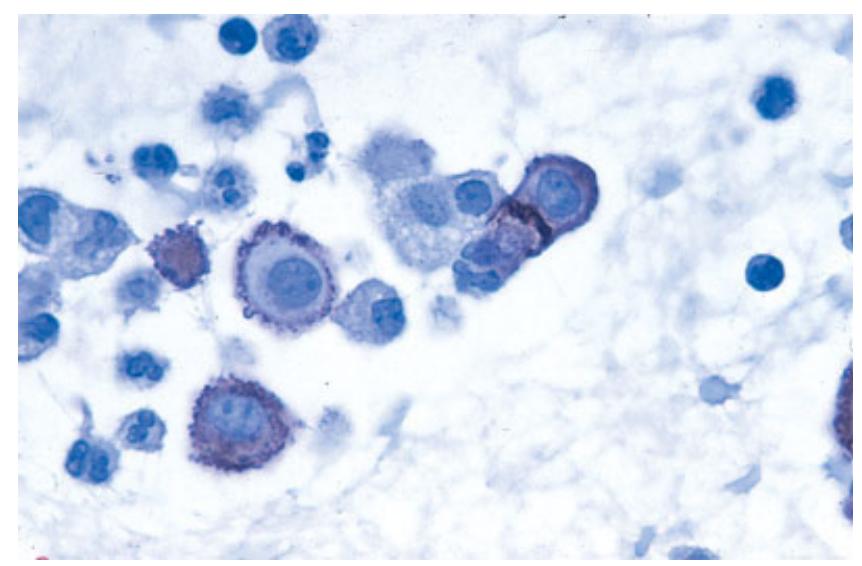

Fig. C-1

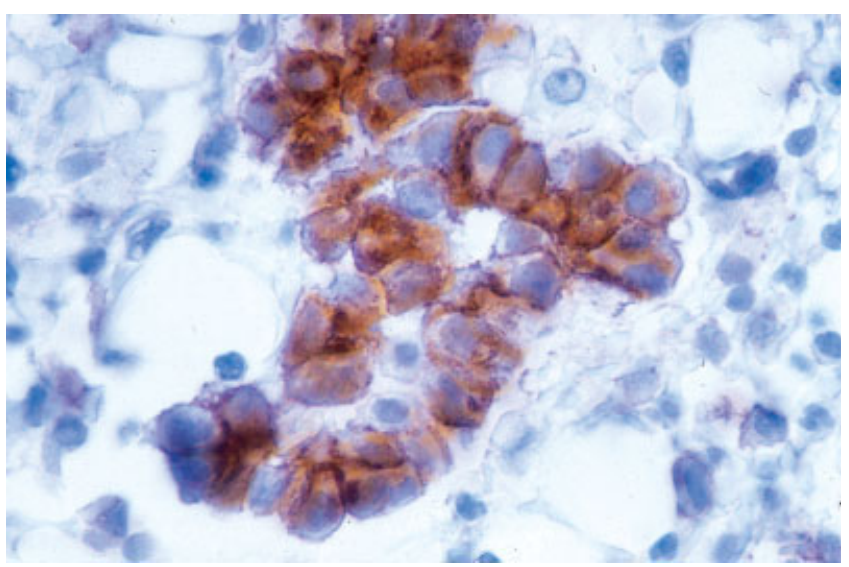

Fig. C-3

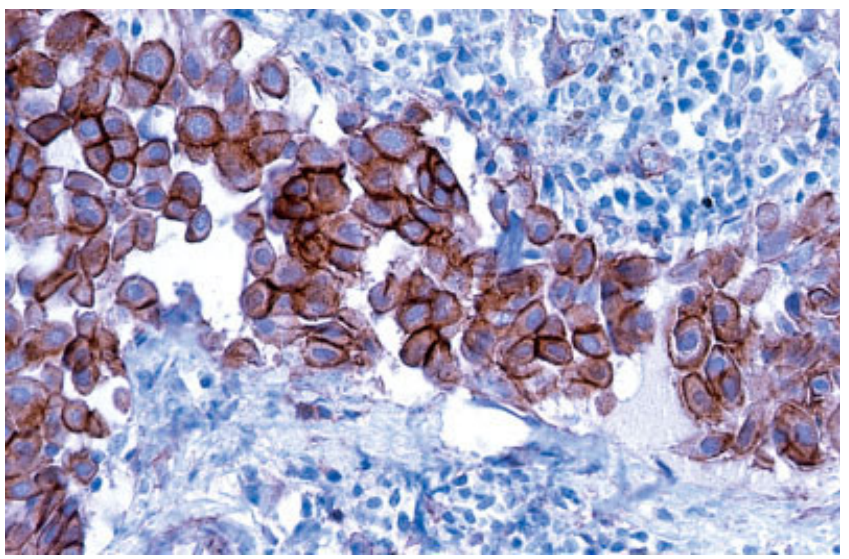

Fig. C-5

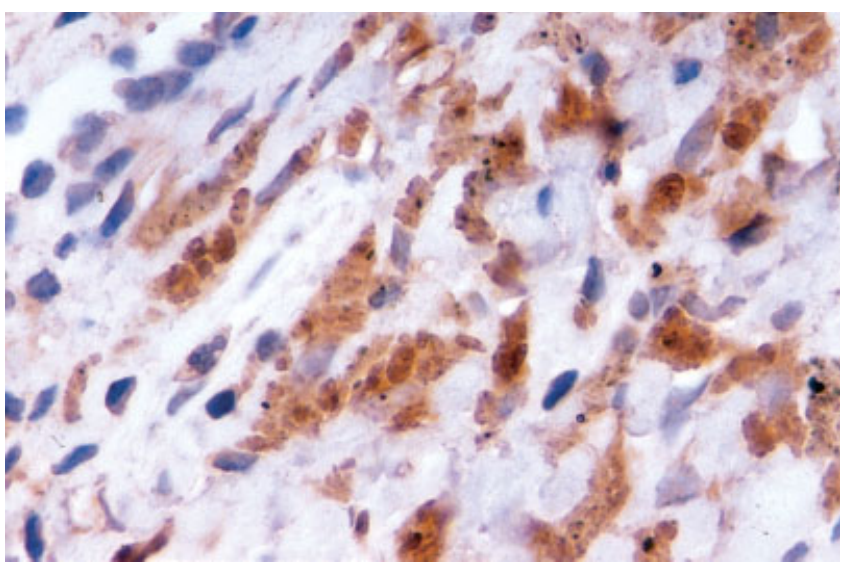

Fig. C-7

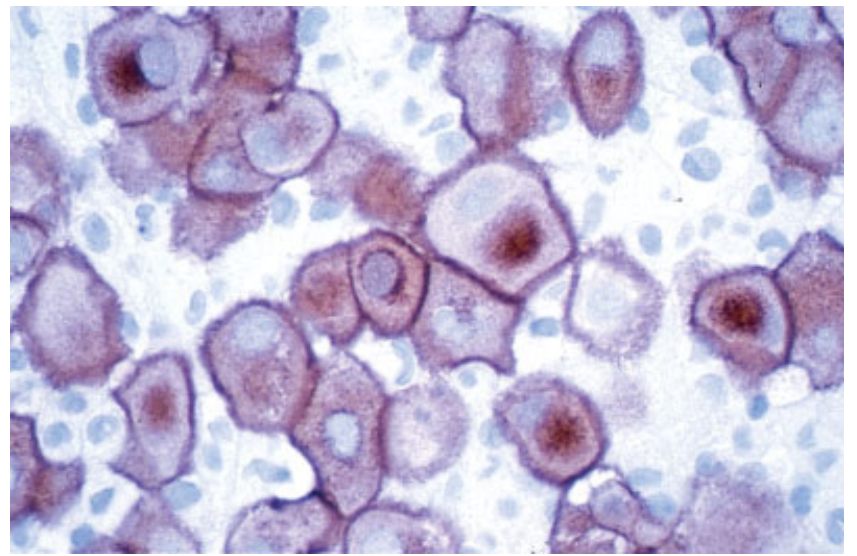

Fig. C-2

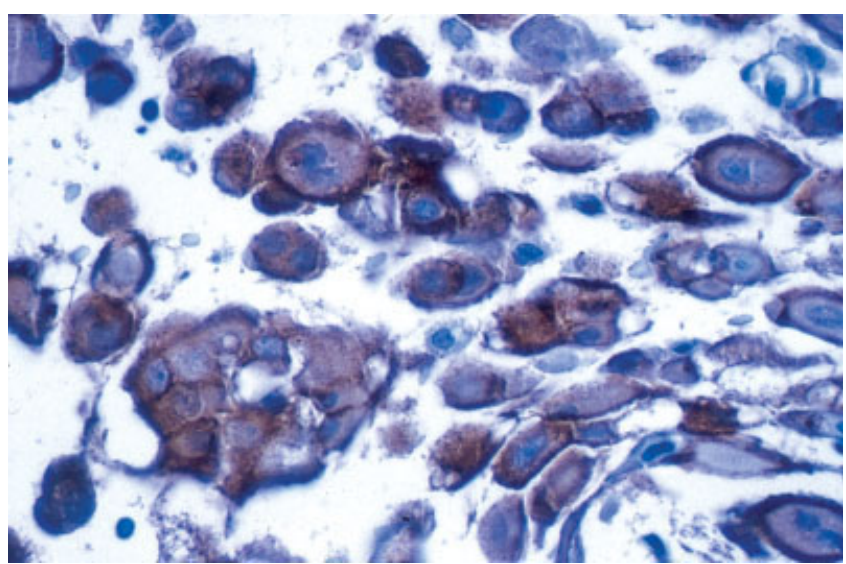

Fig. C-4

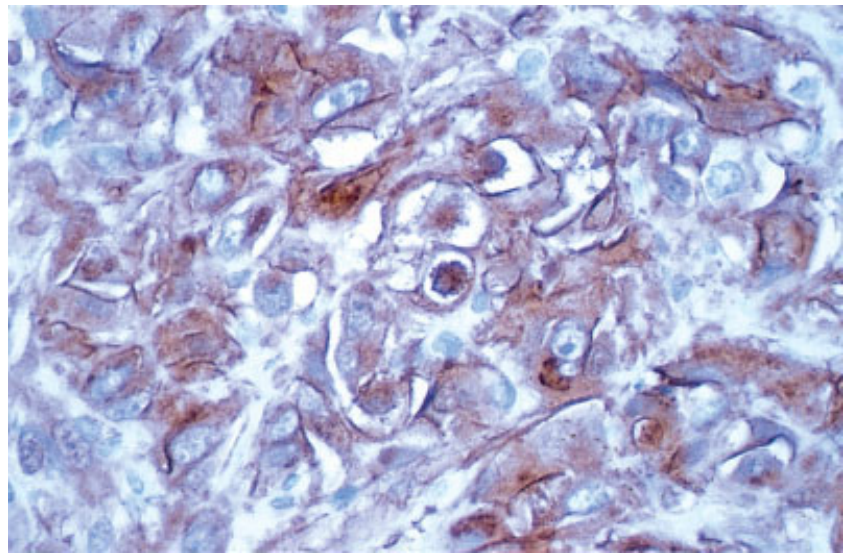

Fig. C-6

Figs. C-1-C-7. Fig. C-1. Reactive mesothelial cells in a cell block showing positive membranous staining for $\beta$-catenin $(\times 100)$. Fig. C-2. Malignan mesothelioma cells in a cell block showing positive membranous and nuclear staining for $\beta$-catenin $(\times 100)$. Fig. C-3. Malignant mesothelioma cells in a cell block showing positive cytoplasmic staining for $\beta$-catenin $(\times 60)$. Fig. C-4. Cells of early mesothelioma in a cell block showing positive cytoplasmic staining for $\beta$-catenin $(\times 60)$. Fig. C-5. Cells of early mesothelioma in a cell block showing positive cytoplasmic staining for $\beta$-catenin $(\times 40)$. Fig. C-6. Deep portion of malignant mesothelioma from a pleural resection showing positive cytoplasmic and nuclear staining for $\beta$-catenin $(\times 100)$. Fig. C-7. Sarcomatoid malignant mesothelioma from a pleural resection showing positive cytoplasmic and nuclear staining for $\beta$-catenin $(\times 40)$. 
Table I. $\beta$-Catenin Expression in Mesothelial Lesions

\begin{tabular}{|c|c|c|c|c|c|}
\hline Lesions & Membranous/submembranous & Cytoplasmic & Nuclear & Cytoplasmic and Nuclear & Total \\
\hline Invasive mesothelioma & $7(21 \%)$ & $14(42 \%)$ & $2(7 \%)$ & $10(30 \%)$ & 33 \\
\hline Early mesothelioma & $2(22 \%)$ & $4(44 \%)$ & $0(0 \%)$ & $3(34 \%)$ & 9 \\
\hline Reactive mesothelium & $20(87 \%)$ & $3(13 \%)$ & $0(0 \%)$ & $0(0 \%)$ & 23 \\
\hline
\end{tabular}

expression of $\beta$-catenin might lead to loss of adhesiveness and increasing invasive and metastatic potential. It has been reported that $100 \%$ of thyroid anaplastic carcinoma, $88 \%$ of head and neck squamous cell carcinomas, $70 \%$ of esophageal carcinomas, and $30 \%$ of invasive ductal carcinomas of the breast show reduced membranous $\beta$-catenin expression. Results of our study are similar: Twenty of the 23 cases $(87 \%)$ in reactive mesothelial lesions showed membranous positivity. Only 7 of 33 (21\%) malignant mesotheliomas were positive for membranous $\beta$-catenin. Two of $9(22 \%)$ early mesotheliomas showed membranous stain for $\beta$-catenin.

In contrast, cytoplasmic over expression of $\beta$-catenin was identified in $24 / 33(72 \%)$ malignant mesothelioma cases, in $7 / 9(78 \%)$ early mesothelioma, and only in $3 / 23$ $(13 \%)$ benign mesothelial hyperplasia cases. Nuclear positivity of $\beta$-catenin was revealed in $10 / 33$ (30\%) malignant mesothelioma and 3/9 (34\%) early mesotheliomas. No nuclear stain of $\beta$-catenin was observed in normal or reactive mesothelium. As many investigators reported, ${ }^{12} \beta$-catenin participates in the transduction of signals and activates transcription by forming complexes with DNA binding proteins in T-cell factor lymphoid enhancer factor (TcF-lef) family. ${ }^{13}$ The free (cytoplasmic and nuclear) $\beta$ catenin level is low in normal cells because APC protein, together with glycogen synthesize kinase-3b (GSK-3b), and other molecules such as axin, clear up unnecessary $\beta$ catenin from the cytoplasm. Mutations that inactivate APC increase cytoplasmic levels of free $\beta$-catenin and in the same way, $\beta$-catenin gene mutations cause overexpression of $\beta$-catenin in cytoplasm and nuclei as well. The $\beta$-catenin accumulation in cytoplasm and nuclei has been shown to be strongly associated with malignant transformation, such as colon cancer, melanoma, hepatocellular carcinoma (HCC), ovarian carcinoma, endometrial cancer, and prostate cancer. ${ }^{14-16}$ Therefore, widespread $\beta$-catenin in the cytoplasm and nucleus may be a potential marker of malignant mesotheliomas. On the basis of our results, the nuclear staining of $\beta$-catenin is more specific in distinguishing mesothelioma from benign mesothelial hyperplasia. Therefore, nuclear staining of $\beta$-catenin has potential application in the separation of cytologically bland malignant mesothelioma from florid mesothelial hyperplasia.

Some studies found that the down-regulation of functional adhesive $\beta$-catenin and over-expression of $\beta$-catenin in cytoplasm and nucleus are associated with the degree of dedifferentiation, and are directly related to the invasiveness of many human epithelial tumors. ${ }^{17}$ However, our result does not reveal a strong association between the degree of dedifferentiation and the degree of $\beta$-catenin over-expression in cytoplasm and nucleus. The nuclear and cytoplasmic positivities of $\beta$-catenin are $3 / 9(34 \%)$ and $7 / 9(78 \%)$ in early mesothelioma (in situ); 10/33(30\%) and $24 / 33(72 \%)$ in invasive malignant mesothelioma. This may be due to the fact that most malignant mesotheliomas in our study are the well-differentiated epithelial types, which do not show the same degree of down- regulation as do other carcinomas.

In summary, this study revealed a distribution pattern of $\beta$-catenin in mesothelial lesions, characterized by decreasing of membranous and/or submembranous staining and increasing of nuclear/cytoplasmic staining with the progression from benign reactive lesions to invasive mesotheliomas. The patterns of $\beta$-catenin expression within the lesions seem to parallel the spectrum of the biologic change seen in these mesothelial proliferative and neoplastic diseases. Identification of $\beta$-catenin staining pattern might offer a useful marker in the diagnosis of mesothelial lesions and help identify neoplastic changes. To our knowledge, this is the first study evaluating the expression of $\beta$-catenin in the spectrum of mesothelial proliferations and cytologic specimens. Additional studies with a larger number of mesotheliomas, ideally with both histologic and cytologic correlation, are needed to further confirm the findings of this study.

\section{References}

1. Pass HI, Robinson BW, Testa JR, Carbone M. Emerging translational therapies for mesothelioma. Chest 1999;116(Suppl. 6):455S460S.

2. Churg A, Colby TV, Cagle P, et al. The separation of benign and malignant mesothelial proliferations. Am J Surg Pathol 2000;24(9): 1183-1200.

3. Morin PJ. Beta-catenin signaling and cancer. Bioessays 1999;21(12): 1021-1030.

4. Ben-Ze'ev A, Shtutman M, Zhurinsky J. The integration of cell adhesion with gene expression: the role of beta-catenin. Exp Cell Res 2000;261(1):75-82.

5. Saegusa M, Okayasu I. Frequent nuclear beta-catenin accumulation and associated mutations in endometrioid-type endometrial and ovarian carcinomas with squamous differentiation. J Pathol 2001;194(1):59-67.

6. Whitaker D, Henderson DW, Shilkin KB. The concept of mesothelioma in situ: implications for diagnosis and histogenesis. Semin Diagn Pathol 1992;9(2):151-161.

7. Chambers HM, Henderson DW. Test and teach. Number twentyseven. Diagnosis: malignant pleural mesothelioma. Pathology 1981; 13(1):8-9,159-161. 
DAI ET AL.

8. Bedrossian CW, Bonsib S, Moran C. Differential diagnosis between mesothelioma and adenocarcinoma: a multimodal approach based on ultrastructure and immunocytochemistry. Semin Diagn Pathol 1992; 9(2):124-140.

9. Bedrossian CW. Diagnostic problems in serous effusions. Diagn Cytopathol 1998;19(2):131-137.

10. McCaughey WT, Al-Jabi M. Differentiation of serosal hyperplasia and neoplasia in biopsies. Pathol Annu 1986;21Pt 1:271-293.

11. Henderson DW, Shilkin KB, Whitaker D. Reactive mesothelial hyperplasia vs mesothelioma, including mesothelioma in situ: a brief review. Am J Clin Pathol 1998;110(3):397-404.

12. Cerrato A, Fulciniti F, Avallone A, Benincasa G, Palombini L, Grieco M. Beta- and gamma-catenin expression in thyroid carcinomas. J Pathol 1998;185(3):267-272.

13. Shtutman M, Zhurinsky J, Simcha I, et al. The cyclin D1 gene is a target of the beta-catenin/LEF-1 pathway. Proc Natl Acad Sci U S A 1999;96(10):5522-5527.
14. Montgomery E, Torbenson MS, Kaushal M, Fisher C, Abraham SC. Beta-catenin immunohistochemistry separates mesenteric fibromatosis from gastrointestinal stromal tumor and sclerosing mesenteritis. Am J Surg Pathol 2002;26(10):1296-1301.

15. Gamallo C, Palacios J, Moreno G, Calvo de Mora J, Suarez A, Armas A. Beta- catenin expression pattern in stage I and II ovarian carcinomas: relationship with beta-catenin gene mutations, clinicopathological features, and clinical outcome. Am J Pathol 1999; 155(2):527-536.

16. Bian YS, Osterheld MC, Bosman FT, Fontolliet C, Benhattar J. Nuclear accumulation of beta-catenin is a common and early event during neoplastic progression of Barrett esophagus. Am J Clin Pathol 2000;114(4):583-590.

17. Birchmeier W, Behrens J. Cadherin expression in carcinomas: role in the formation of cell junctions and the prevention of invasiveness. Biochim Biophys Acta 1994;1198(1):11-26. 